\title{
Characterizing New Eclipsing Binaries Identified from STEREO Photometry
}

\author{
Harry Markov ${ }^{1}$, Zlatan Tsvetanov ${ }^{2}$, \\ Ilian Iliev ${ }^{1}$, Ivanka Stateva ${ }^{1}$ and Nevena Markova ${ }^{1}$ \\ ${ }^{1}$ Institut of Astronomy and National Astronomical Observatory Rozhen, Bulgaria, \\ ${ }^{2}$ Johns Hopkins University, Department of Physics and Astronomy, USA \\ email: hmarkov@astro.bas.bg
}

\begin{abstract}
Since 2010, a program to explore new eclipsing binary systems identified from STEREO photometry has been in progress. Our first results are presented here: light curves and high resolution spectra taken with Coudé spectrograph (National Astronomical Observatory Rozhen) and ARC Échelle spectrometer (ARCES, Apache Point Observatory).
\end{abstract}

Keywords. techniques: radial velocities, photometric, spectroscopic; binaries: eclipsing

\section{Introduction}

STEREO is a mission in the NASA Solar Terrestrial Probes program. It uses two nearly identical spacecraft to map coronal mass ejections as they propagate away from the Sun. The continuous series of images obtained by the Heliospheric Imager 1 (HI-1) cameras on the two STEREO S/Cs are well-suited to detect variations in the brightness of the light sources in the explored fields. The STEREO HI-1 photometry provides a complete survey of all bright stars $(<10 \mathrm{mag})$ for $18 \%$ of the sky and our project is aimed at detecting transiting exoplanets. As a natural by-product, a substantial number of EBs are detected. In the course of our STEREO project, we obtained and examined aperture photometry of isolated stars extracted from the HI-1A images for four years worth of data (2007-2010). Our input catalogue includes over 70,000 Tycho 2 stars. This coarse examination revealed over $250 \mathrm{EBs}$, with fully half of them being new. Many of these EBs were recently independently reported by Wright et al. (2011), and we note here that our EB list includes about $10 \%$ more objects.

Recent available EB models, combined with radial velocity (RV) measurements, can provide a wealth of useful astrophysical information. The goal of our program is to supplement available STEREO photometric light curves with accurate RV curves. For this purpose, high resolution spectroscopic observations are already in progress with the Coude spectrograph $(\mathrm{R}=15000$ and $\mathrm{R}=30000)$ of the National Astronomical Observatory Rozhen (Bulgaria) and the ARC Echelle spectrometer (ARCES, R=30000) of the Apache Point Observatory (New Mexico, USA). RV measurements were derived through the cross-correlation method, with a mean standard deviation of about $1.5 \mathrm{~km} / \mathrm{sec}$ estimated by using different template spectra from the Montes et al. (1997) library and RV stable stars taken on the observing night. Particular attention will be paid to systems including small components (late $\mathrm{K}$ and $\mathrm{M}$ dwarfs) since the observational uncertainties in this part of the stellar mass function are the largest.

\section{Results and notes on individual objects}

In Fig. 1, we demonstrate the light curves for some EB systems derived from STEREO observations. The data presented here are photometry time series extracted from HI-1A images from four years of observing (2007-2010). We use the latest available calibration 

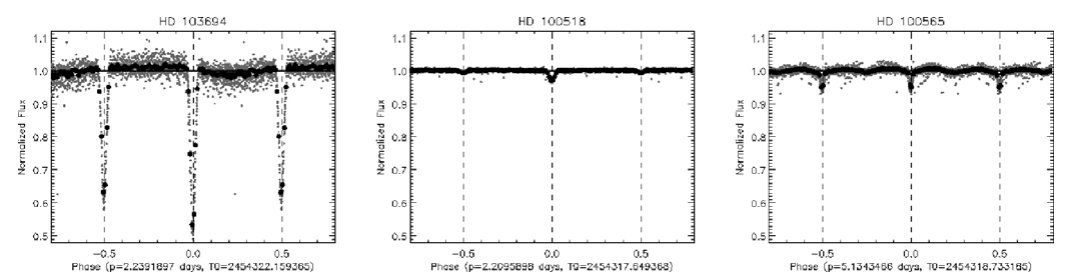

Figure 1. STEREO photometry light curves of some EB systems.

from the STEREO pipeline plus additional calibration developed by us. Low frequency trends were taken out by fitting low order polynomials. The stars shown here were our first targets for the follow-up spectroscopic observations.

HD103694 (see Fig. 2) shows double lined spectra (early to late) which allowed us to derive the separate RV curves of the components and their physical and kinematic characteristics, assuming circular orbits.
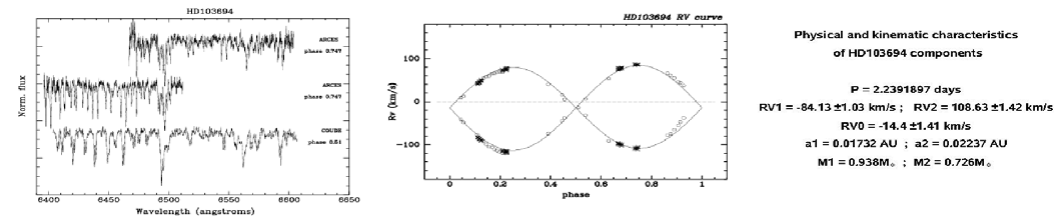

Figure 2. Spectra (left) and RV curves (center) of both HD103694 components. Some physical and kinematic characteristics are shown on the right

HD100565 (Fig. 3, left panel): The spectral lines measured on two spectra are shifted by about $54 \mathrm{~km} / \mathrm{s}$ in less than 24 hours. This clearly shows the double nature of this system. Although the photometric light curve (Fig.1 above) does not demonstrate strong evidence of a second component, we announce HD100565 as a newly found SB1 type EBS.

HD100518 (HR4454) (Fig. 3, right panel): The spectra show at least two different spectral line sets. Measurements of the stronger one revealed relatively low velocities shifts $(\sim 3 \mathrm{~km} / \mathrm{s})$ with the phase, but the weaker one (marked by vertical lines) showed significant displacement. Griffin (2006) suspected the system is a multiple. Here, for the first time, we show the spectral features of another component. The STEREO light curve revealed the presence of an EB component with a new period $(2.2 \mathrm{~d})$ different from those measured by Griffin (2006) in his extensive RV study.
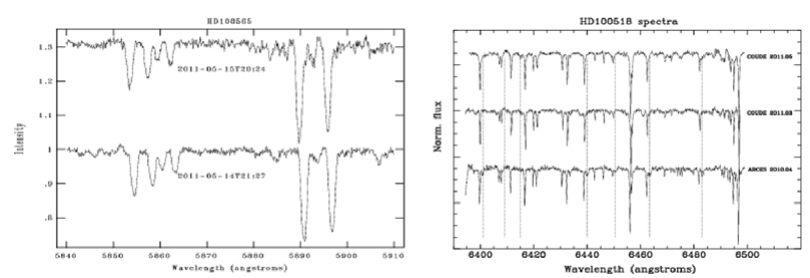

Figure 3. Spectra of HD100565 (left) and HD100518 (=HR4454) (right).

Acknowledgements: This work is supported by the Bulgarian National Science Fund grant DO 02-85, and also NASA grant NNX10AM33G (ZT). This work made use of the SIMBAD and VIZIER data bases, operated at CDS, Strasbourg, France.

\section{References}

Griffin R. F. 2006, Observatory, 126, 1

Montes D., Martin E. L., Fernandez-Figueroa M. J., et al., 1997, A\&AS, 123, 473

Wright, K. T. et al., 2011, arXiv:1103.0911 